\title{
A Fourth Order Finite Difference Method for Singularly Perturbed Differential-Difference Equations
}

\author{
R. Nageshwar Rao, P. Pramod Chakravarthy"
}

Department of Mathematics, Visvesvaraya National Institute of Technology, Nagpur, 440010, India

\begin{abstract}
This paper deals with the singularly perturbed boundary value problem for a linear second order differential-difference equation of the convection-diffusion type with small delay parameter. A fourth order finite difference method is developed for solving singularly perturbed differential difference equations. To handle the delay argument, we construct a special type of mesh, so that the term containing delay lies on nodal points after discretization. The proposed finite difference method works nicely when the delay parameter is smaller or bigger to perturbation parameter. The truncation error of the finite difference method is calculated. On the basis of truncation error, as well as the results of number of computational examples, it is concluded that the present method offers significant advantage for the linear problems.
\end{abstract}

Keywords Differential-Difference Equation, Singularly-Perturbed, Boundary Layer, Oscillations, Delay, Finite Difference Scheme

\section{Introduction}

A singularly perturbed differential-difference equation is an ordinary differential equation in which the highest derivative is multiplied by a small parameter and involving at least one delay term. Such problems are found throughout the literature on epidemics and population dynamics where these small shifts play an important role in the modelling of various real life phenomena [1]. Boundary value problems in differential-difference equations arise in a very natural way in studying variational problems in control theory where the problem is complicated by the effect of time delays in signal transmission[2]. In the mathematical model for the determination of the expected first-exit time in the generation of action potential in nerve cells by random synaptic inputs in dendrites, the shifts are due to the jumps in the potential membrane which are very small[3].

Lange and Miura[4] gave an asymptotic approach in the study of a class of boundary value problems for linear second order differential-difference equations in which the highest order derivative is multiplied by a small parameter. It has been shown that the layer behavior can change its character and even be destroyed as the shifts increase but remain small. In the companion paper[5], similar boundary value problems with solutions that exhibit rapid oscillations are studied. An extensive numerical work had been initiated by M. K. Kadalbajoo and K. K. Sharma in their papers[6-8]. In

* Corresponding author:

pramodpodila@yahoo.co.in (P. Pramod Chakravarthy )

Published online at http://journal.sapub.org/ajcam

Copyright (C) 2011 Scientific \& Academic Publishing. All Rights Reserved
[6], the authors proposed a numerical method for boundary value problems for singularly perturbed differential- difference equations with a negative shift. Here the shifted term is approximated by Taylor series and a difference scheme is applied. This method works provided the shift is of o $(\varepsilon)$. In[7], a numerical method based on the fitted mesh approach to approximate the solution of these types of boundary value problems is presented. Here the piecewise uniform meshes are constructed and fitted to the boundary layer regions to adapt singular behaviour of the operator in these narrow regions. $\operatorname{In}[8]$, the authors proposed a numerical method to solve the boundary value problems for singularly perturbed delay differential equations, which work nicely in both the cases, i.e., the delay argument is bigger as well as smaller than the perturbation parameter. To handle the delay argument, they constructed a special type of mesh so that the term containing delay lies on nodal points after discretization. In[11], the authors M.K. Kadalbajoo and Devendra Kumar presented a numerical method for boundary value problems for nonlinear singularly perturbed differential-difference equations with small delay. Here Quasilinearization process is used to linearize the nonlinear differential equation. After applying the quasilinearization process to the nonlinear problem, a sequence of linearized problems is obtained. A piecewise-uniform mesh is used, which is dense in the boundary layer region and coarse in the outer region. $\operatorname{In}[12]$, the authors Gabil M. Amiraliyev, Erkan Cimen had given a numerical method for singularly perturbed boundary value problem for a linear second order delay differential equation with a large delay in the reaction term. The authors presented an exponentially fitted difference scheme on a uniform mesh which is accomplished by the method of integral identities with the use of exponential basis functions and interpolating 
quadrature rules with weight and remainder term in integral form. In[13], the authors M.K. Kadalbajoo, Devendra Kumar presented a numerical method for singularly perturbed boundary value problem for a linear second order differential-difference equation of the convection-diffusion type with small delay parameter. Taylor series is used to tackle the delay term. The fitted mesh technique is employed to generate a piecewise-uniform mesh, condensed in the neighbourhood of the boundary layers. B-spline collocation method is used with fitted mesh. In[14], the authors M.K. Kadalbajoo, V.P. Ramesh presented a hybrid algorithm for singularly perturbed second order differential difference equation of the convection diffusion type with a small delay. The convection term with the delay is expanded in Taylor series and numerical schemes on Shishkin mesh are constructed. In[15], the authors Jugal Mohapatra, Srinivasan Natesan constructed a numerical method for a class of singularly perturbed differential-difference equations with small delay. The numerical method comprises of upwind finite difference operator on an adaptive grid, which is formed by equi distributing the arc-length monitor function.

In this paper a fourth order finite difference scheme is developed for solving singularly perturbed differential-difference equations. To handle the delay argument, we construct a special type of mesh, so that the term containing delay lies on nodal points after discretization. The proposed finite difference method works nicely when the delay parameter is smaller or bigger to perturbation parameter. The truncation error of the finite difference scheme is calculated. On the basis of truncation error, as well as the results of number of computational examples, it is concluded that the present method offers significant advantage for the linear problems.

\section{Fourth Order Finite Difference Method}

To describe the fourth order finite difference method, we consider a linear singularly perturbed differential-difference equation, which contains only negative shift in the convection term

$$
\varepsilon y^{\prime \prime}(x)+a(x) y^{\prime}(x-\delta)+b(x) y(x)=f(x)
$$

on $0<\mathrm{x}<1,0<\varepsilon<<1$, subject to the interval and boundary conditions

$$
\begin{aligned}
& y(x)=\varphi(x) ;-\delta \leq x \leq 0 \\
& y(1)=\beta
\end{aligned}
$$

where $a(x), b(x), f(x)$ and $\varphi(x)$ are known analytic functions and, further, that each function is simple enough so that analytic differentiation is feasible, $\beta$ is a constant and $\delta(\varepsilon)$ is a small shifting parameter. For a function $\mathrm{y}(\mathrm{x})$ to constitute a smooth solution of (1)-(2) it must be continuous on $[0,1]$ and be continuously differentiable on $(0,1)$. For $\delta=0$ the corresponding ordinary differential equation has solutions with a layer on the left or on the right for $\mathrm{a}(\mathrm{x})>0$ or $\mathrm{a}(\mathrm{x})<0$ on $0 \leq \mathrm{x} \leq 1$, respectively. The layer is maintained at the same end for $\delta$ sufficiently small i.e., $\delta=\mathrm{o}(\varepsilon)$. The layer behaviour can change its character and even be destroyed as the shifts increase, i.e., when $\delta=\mathrm{O}(\varepsilon)[4]$.

To handle the delay argument, we construct a special type of mesh, so that the term containing delay lies on nodal points after discretization. We divide the interval $[0,1]$ in to $\mathrm{N}$ equal parts by choosing the mesh parameter $\mathrm{h}=\delta / \mathrm{m}$, where $\mathrm{m}$ is a positive integer chosen such that $1<\mathrm{m}<\mathrm{N}$.

Using central difference formulae, the finite difference representation of equation (1) may be written at a typical mesh point $\mathrm{x}_{\mathrm{i}}, \mathrm{i}=0,1, \ldots, \mathrm{N}$, as

$$
\begin{gathered}
\frac{\varepsilon}{\mathrm{h}^{2}}\left\{\delta^{2}-\frac{1}{12} \delta^{4}\right\} \mathrm{y}_{\mathrm{i}}+\frac{\mathrm{a}_{\mathrm{i}}}{\mathrm{h}}\left\{\mu \delta-\frac{1}{6} \mu \delta^{3}\right\} \mathrm{y}_{\mathrm{i}-\mathrm{m}}+\mathrm{b}_{\mathrm{i}} \mathrm{y}_{\mathrm{i}}= \\
\mathrm{f}_{\mathrm{i}}+\mathrm{B}_{\mathrm{i}} \mathrm{y}_{\mathrm{i}}+\mathrm{C}_{\mathrm{i}} \mathrm{y}_{\mathrm{i}-\mathrm{m}}
\end{gathered}
$$

where

$$
\mathrm{B}_{\mathrm{i}}=-\frac{\varepsilon}{\mathrm{h}^{2}}\left\{\frac{1}{90} \delta^{6}+\ldots\right\} \text { and } \mathrm{C}_{\mathrm{i}}=-\frac{\mathrm{a}_{\mathrm{i}}}{\mathrm{h}}\left\{\frac{1}{30} \mu \delta^{5}+\ldots\right\}
$$

and $a\left(x_{i}\right)=a_{i} ; b\left(x_{i}\right)=b_{i} ; f\left(x_{i}\right)=f_{i} ; y\left(x_{i}\right)=y_{i}$ and $\delta$ is the standard central difference operator, $\mu$ is the averaging operator[16].

The boundary conditions are

$$
\mathrm{y}_{\mathrm{i}}=\phi_{\mathrm{i}}, \quad \text { for } \mathrm{i} \text { a non positive integer } \mathrm{y}_{\mathrm{N}}=\beta
$$

where $\varphi_{\mathrm{i}}=\varphi\left(\mathrm{x}_{\mathrm{i}}\right)$.

The left side of equation (3) involves the finite differences $\delta^{4} y_{i}$ and $\mu \delta^{3} y_{i-m}$. We approximate $\mu \delta^{3} y_{i-m}, \delta^{4} y_{i}$ correct to $\mathrm{O}\left(\mathrm{h}^{5}\right)$ and $\mathrm{O}\left(\mathrm{h}^{6}\right)$, respectively as follows:

Differentiating (1) once with respect to $\mathrm{x}$, then using central difference formulae, gives a $\mathrm{O}\left(\mathrm{h}^{5}\right)$ approximation for $\mu \delta^{3} y_{i-m}$ as follows :

$$
\begin{aligned}
& \mu \delta^{3} \mathrm{y}_{\mathrm{i}-\mathrm{m}}=\frac{\mathrm{h}^{3}}{\varepsilon} \mathrm{f}_{\mathrm{i}-\mathrm{m}}^{\prime}-\frac{\mathrm{ha} \mathrm{i}_{\mathrm{i}-\mathrm{m}}}{\varepsilon} \delta^{2} \mathrm{y}_{\mathrm{i}-2 \mathrm{~m}}-\frac{\mathrm{h}^{2}}{\varepsilon} \mathrm{a}_{\mathrm{i}-\mathrm{m}}^{\prime} \mu \delta \mathrm{y}_{\mathrm{i}-2 \mathrm{~m}} \\
& -\frac{\mathrm{h}^{2}}{\varepsilon} \mathrm{b}_{\mathrm{i}-\mathrm{m}} \mu \delta \mathrm{y}_{\mathrm{i}-\mathrm{m}}-\frac{\mathrm{h}^{3}}{\varepsilon} \mathrm{b}_{\mathrm{i}-\mathrm{m}}^{\prime} \mathrm{y}_{\mathrm{i}-\mathrm{m}}+\mathrm{D}_{\mathrm{i}} \mathrm{y}_{\mathrm{i}-\mathrm{m}}+\mathrm{E}_{\mathrm{i}} \mathrm{y}_{\mathrm{i}-2 \mathrm{~m}}
\end{aligned}
$$

where

$$
\begin{aligned}
\mathrm{D}_{\mathrm{i}}= & \frac{1}{4} \mu \delta^{5}+\ldots+\frac{\mathrm{h}^{2}}{6 \varepsilon} \mathrm{b}_{\mathrm{i}-\mathrm{m}} \mu \delta^{3}-\frac{\mathrm{h}^{2}}{30 \varepsilon} \mathrm{b}_{\mathrm{i}-\mathrm{m}} \mu \delta^{5} \ldots \\
E_{i} & =\frac{h}{12 \varepsilon} a_{i-m} \delta^{4}-\frac{h}{90 \varepsilon} a_{i-m} \delta^{6} \ldots+\frac{h^{2}}{6 \varepsilon} a_{i-m}^{\prime} \mu \delta^{3} \\
& -\frac{h^{2}}{30 \varepsilon} a_{i-m}^{\prime} \mu \delta^{5} \ldots
\end{aligned}
$$

and $a^{\prime}\left(x_{i}\right)=a_{i}^{\prime} ; b^{\prime}\left(x_{i}\right)=b_{i}^{\prime} ; f^{\prime}\left(x_{i}\right)=f_{i}^{\prime} ; y^{\prime}\left(x_{i}\right)=y_{i}^{\prime} \quad$ Similarly differentiating (1) twice, then using central difference formulae, gives a $\mathrm{O}\left(\mathrm{h}^{6}\right)$ approximation for $\delta^{4} y_{\mathrm{i}}$ as follows:

$$
\begin{aligned}
& \delta^{4} y_{i}=\frac{h^{4}}{\varepsilon} f_{i}^{\prime \prime}-\frac{h^{4}}{\varepsilon^{2}} a_{i} f_{i-m}^{\prime}+\frac{h^{2}}{\varepsilon^{2}} a_{i} a_{i-m} \delta^{2} y_{i-2 m}+\frac{h^{3}}{\varepsilon^{2}} a_{i} a_{i-m}^{\prime} \mu \delta y_{i-2 m} \\
& +\frac{h^{3}}{\varepsilon^{2}} a_{i} b_{i-m} \mu \delta y_{i-m}+\frac{h^{4}}{\varepsilon^{2}} a_{i} b_{i-m}^{\prime} y_{i-m}-\frac{2 h^{2}}{\varepsilon} a_{i}^{\prime} \delta^{2} y_{i-m} \\
& -\frac{h^{3}}{\varepsilon} a_{i}^{\prime \prime} \mu \delta y_{i-m}-\frac{h^{2}}{\varepsilon} b_{i} \delta^{2} y_{i}-\frac{2 h^{3}}{\varepsilon} b_{i}^{\prime} \mu \delta y_{i}-\frac{h^{4}}{\varepsilon} b_{i}^{\prime \prime} y_{i}- \\
& \frac{h}{\varepsilon} a_{i} D_{i} y_{i-m}-\frac{h}{\varepsilon} a_{i} E_{i} y_{i-2 m}+G_{i} y_{i}+H_{i} y_{i-m}
\end{aligned}
$$




$$
\begin{aligned}
& \mathrm{G}_{\mathrm{i}}=\frac{1}{6} \delta^{6}+\ldots+\frac{\mathrm{h}^{2}}{12 \varepsilon} \mathrm{b}_{\mathrm{i}} \delta^{4}-\frac{\mathrm{h}^{2}}{90 \varepsilon} \mathrm{b}_{\mathrm{i}} \delta^{6} \ldots+ \\
& \frac{\mathrm{h}^{3}}{3 \varepsilon} \mathrm{b}_{\mathrm{i}}^{\prime} \mu \delta^{3}-\frac{\mathrm{h}^{3}}{15 \varepsilon} \mathrm{b}_{\mathrm{i}}^{\prime} \mu \delta^{5} \ldots \\
& \mathrm{H}_{\mathrm{i}}=\frac{\mathrm{h}}{4 \varepsilon} \mathrm{a}_{\mathrm{i}} \mu \delta^{5}+\ldots+\frac{\mathrm{h}^{2}}{6 \varepsilon} \mathrm{a}_{\mathrm{i}}^{\prime} \delta^{4}-\frac{\mathrm{h}^{2}}{45 \varepsilon} \mathrm{a}_{\mathrm{i}}^{\prime} \delta^{6}+\ldots+ \\
& \frac{\mathrm{h}^{3}}{6 \varepsilon} \mathrm{a}_{\mathrm{i}}^{\prime \prime} \mu \delta^{3}-\frac{\mathrm{h}^{3}}{30 \varepsilon} \mathrm{a}_{\mathrm{i}}^{\prime \prime} \mu \delta^{5} \ldots
\end{aligned}
$$

Substituting (6) and (9) in (3) and simplifying, we get the fourth order finite difference scheme as

where

$$
\begin{aligned}
& p_{i} y_{i+1}+q_{i} y_{i}+r_{i} y_{i-1}+u_{i} y_{i-m+1}+v_{i} y_{i-m} \\
& +w_{i} y_{i-m-1}+\xi_{i} y_{i-2 m+1}+\eta_{i} y_{i-2 m}+ \\
& \zeta_{i} y_{i-2 m-1}=R_{i}+\widetilde{E}_{i} y_{i}+\widetilde{F}_{i} y_{i-m}+\widetilde{G}_{i} y_{i-2 m}
\end{aligned}
$$

$$
\begin{aligned}
& \mathrm{p}_{\mathrm{i}}=\frac{\varepsilon}{\mathrm{h}^{2}}+\frac{1}{12} \mathrm{~b}_{\mathrm{i}}+\frac{\mathrm{h}}{12} \mathrm{~b}_{\mathrm{i}}^{\prime}, \\
& \mathrm{q}_{\mathrm{i}}=-\frac{2 \varepsilon}{\mathrm{h}^{2}}+\frac{5}{6} \mathrm{~b}_{\mathrm{i}}+\frac{\mathrm{h}^{2}}{12} \mathrm{~b}_{\mathrm{i}}^{\prime \prime}, \\
& \mathrm{r}_{\mathrm{i}}=\frac{\varepsilon}{\mathrm{h}^{2}}+\frac{1}{12} \mathrm{~b}_{\mathrm{i}}-\frac{\mathrm{h}}{12} \mathrm{~b}_{\mathrm{i}}^{\prime}, \\
& \mathrm{u}_{\mathrm{i}}=\frac{\mathrm{h}}{24 \varepsilon} \mathrm{a}_{\mathrm{i}} \mathrm{b}_{\mathrm{i}-\mathrm{m}}+\frac{\mathrm{a}_{\mathrm{i}}^{\prime}}{6}+\frac{\mathrm{h}}{24} \mathrm{a}_{\mathrm{i}}^{\prime \prime}+\frac{1}{2 \mathrm{~h}} \mathrm{a}_{\mathrm{i}}, \\
& \mathrm{v}_{\mathrm{i}}=\frac{\mathrm{h}^{2}}{12 \varepsilon} \mathrm{a}_{\mathrm{i}} \mathrm{b}_{\mathrm{i}-\mathrm{m}}^{\prime}-\frac{1}{3} \mathrm{a}_{\mathrm{i}}^{\prime}, \\
& \mathrm{w}_{\mathrm{i}}=-\frac{\mathrm{h}}{24 \varepsilon} \mathrm{a}_{\mathrm{i}} \mathrm{b}_{\mathrm{i}-\mathrm{m}}+\frac{1}{6} \mathrm{a}_{\mathrm{i}}^{\prime}-\frac{\mathrm{h}}{24} \mathrm{a}_{\mathrm{i}}^{\prime \prime}-\frac{1}{2 \mathrm{~h}} \mathrm{a}_{\mathrm{i}}, \\
& \xi_{\mathrm{i}}=\frac{1}{12 \varepsilon} \mathrm{a}_{\mathrm{i}} \mathrm{a}_{\mathrm{i}-\mathrm{m}}+\frac{\mathrm{h}}{24 \varepsilon} \mathrm{a}_{\mathrm{i}} \mathrm{a}_{\mathrm{i}-\mathrm{m}}^{\prime}, \\
& \eta_{\mathrm{i}}=-\frac{1}{6 \varepsilon} \mathrm{a}_{\mathrm{i}} \mathrm{a}_{\mathrm{i}-\mathrm{m}}, \zeta_{\mathrm{i}}=\frac{1}{12 \varepsilon} \mathrm{a}_{\mathrm{i}} \mathrm{a}_{\mathrm{i}-\mathrm{m}}-\frac{\mathrm{h}}{24 \varepsilon} \mathrm{a}_{\mathrm{i}} \mathrm{a}_{\mathrm{i}-\mathrm{m}}^{\prime}, \\
& \mathrm{R}_{\mathrm{i}}=\mathrm{f}_{\mathrm{i}}+\frac{\mathrm{h}^{2}}{12} \mathrm{f}_{\mathrm{i}}^{\prime \prime}+\frac{\mathrm{h}^{2}}{12 \varepsilon} \mathrm{a}_{\mathrm{i}} \mathrm{f}_{\mathrm{i}-\mathrm{m}}^{\prime} .
\end{aligned}
$$

The leading order error terms in equation (12) are given by

$$
\begin{aligned}
\widetilde{\mathrm{E}}_{\mathrm{i}}=\left(\frac{\varepsilon}{360 \mathrm{~h}^{2}}-\frac{\mathrm{b}_{\mathrm{i}}}{1080}\right) \delta^{6}+\ldots-\frac{\mathrm{h}}{180} \mathrm{~b}_{\mathrm{i}}^{\prime} \mu \delta^{5}+\ldots \\
\quad . .+\frac{\mathrm{h}}{36} \mathrm{~b}_{\mathrm{i}}^{\prime} \mu \delta^{3} \ldots \\
\widetilde{\mathrm{F}}_{\mathrm{i}}=-\frac{1}{540} \mathrm{a}_{\mathrm{i}}^{\prime} \delta^{6}+\ldots \\
+\left(-\frac{1}{80 \mathrm{~h}} \mathrm{a}_{\mathrm{i}}+\frac{\mathrm{h}}{360 \varepsilon} \mathrm{a}_{\mathrm{i}} \mathrm{b}_{\mathrm{i}-\mathrm{m}}-\frac{\mathrm{h}}{360} \mathrm{a}_{\mathrm{i}}^{\prime \prime}\right) \mu \delta^{5}+\ldots \\
+\left(-\frac{\mathrm{h}}{72 \varepsilon} \mathrm{a}_{\mathrm{i}} \mathrm{b}_{\mathrm{i}-\mathrm{m}}+\frac{\mathrm{h}}{72} \mathrm{a}_{\mathrm{i}}^{\prime \prime}\right) \mu \delta^{3}+\ldots \\
\widetilde{\mathrm{G}}_{\mathrm{i}}=\frac{1}{1080 \varepsilon} \mathrm{a}_{\mathrm{i}} \mathrm{a}_{\mathrm{i}-\mathrm{m}} \delta^{6}+\ldots \\
+\left(-\frac{\mathrm{a}_{\mathrm{i}}}{48 \mathrm{~h}}+\frac{\mathrm{h}}{36 \varepsilon} \mathrm{a}_{\mathrm{i}} \mathrm{a}_{\mathrm{i}-\mathrm{m}}^{\prime}\right) \mu \delta^{5}+\ldots \\
-\frac{1}{144 \varepsilon} \mathrm{a}_{\mathrm{i}} \mathrm{a}_{\mathrm{i}-\mathrm{m}} \delta^{4}+\ldots-\frac{\mathrm{h}}{72 \varepsilon} \mathrm{a}_{\mathrm{i}} \mathrm{a}_{\mathrm{i}-\mathrm{m}}^{\prime} \mu \delta^{3} \ldots
\end{aligned}
$$

The error term associated with equation (12) is $\mathrm{O}\left(\mathrm{h}^{6}\right)$ and it has been observed that the proposed finite difference method is of order four.

Using (5), the difference scheme (12) can be written as

$$
\begin{gathered}
p_{i} y_{i+1}+q_{i} y_{i}+r_{i} y_{i-1}=R_{i}-u_{i} \varphi_{i-m+1} \\
-v_{i} \varphi_{i-m}-w_{i} \varphi_{i-m-1}-\xi_{i} \varphi_{i-2 m+1}- \\
\eta_{i} \varphi_{i-2 m}-\zeta_{i} \varphi_{i-2 m-1} \\
\text { for } 1 \leq i \leq m-1 \\
p_{i} y_{i+1}+q_{i} y_{i}+r_{i} y_{i-1}+u_{i} y_{i-m+1}= \\
R_{i}-v_{i} \varphi_{i-m}-w_{i} \varphi_{i-m-1}-\xi_{i} \varphi_{i-2 m+1} \\
-\eta_{i} \varphi_{i-2 m}-\zeta_{i} \varphi_{i-2 m-1} \\
p_{i} y_{i+1}+q_{i} y_{i}+r_{i} y_{i-1}+u_{i} y_{i-m+1} \\
+v_{i} y_{i-m}=R_{i}-w_{i} \varphi_{i-m-1}- \\
\xi_{i} \varphi_{i-2 m+1}-\eta_{i} \varphi_{i-2 m}-\zeta_{i} \varphi_{i-2 m-1} \\
p_{i} y_{i+1}+q_{i} y_{i}+r_{i} y_{i-1}+u_{i} y_{i-m+1}+ \\
v_{i} y_{i-m}+w_{i} y_{i-m-1}=\quad \text { for } i=m+1 \\
R_{i}-\xi_{i} \varphi_{i-2 m+1}-\eta_{i} \varphi_{i-2 m}-\zeta_{i} \varphi_{i-2 m-1} \\
\quad \text { for } m+2 \leq i \leq 2 m-1 \\
p_{i} y_{i+1}+q_{i} y_{i}+r_{i} y_{i-1}+u_{i} y_{i-m+1} \\
+v_{i} y_{i-m}+w_{i} y_{i-m-1}+\xi_{i} y_{i-2 m+1}= \\
R_{i}-\eta_{i} \varphi_{i-2 m}-\zeta_{i} \varphi_{i-2 m-1} \\
p_{i} y_{i+1}+q_{i} y_{i}+r_{i} y_{i-1}+u_{i} y_{i-m+1}+ \\
v_{i} y_{i-m}+w_{i} y_{i-m-1}+\xi_{i} y_{i-2 m+1}+ \\
\eta_{i} y_{i-2 m}=R_{i}-\zeta_{i} \varphi_{i-2 m-1} \\
p_{i} y_{i+1}+q_{i} y_{i}+r_{i} y_{i-1}+u_{i} y_{i-m+1} \quad \text { for } i=2 m+1 \\
+v_{i} y_{i-m}+w_{i} y_{i-m-1}+\xi_{i} y_{i-2 m+1} \\
+\eta_{i} y_{i-2 m}+\zeta_{i} y_{i-2 m-1}=R_{i} \\
\text { for } 2 m+2 \leq i \leq n-1
\end{gathered}
$$

The above system of equations is solved by Gauss elimination method with partial pivoting. In fact, any numerical method or analytical method can be used.

\section{Numerical Results}

To demonstrate the applicability of the method we consider three boundary value problems of singularly perturbed linear differential difference equations exhibiting boundary layer at the left end of the interval[0,1], and two problems exhibiting boundary layer at the right end of the under lying interval. These examples were discussed in $[4,5,8]$. Since the exact solutions of the problems for different values of $\delta$ are not known, the maximum absolute errors for the examples are calculated using the following double mesh principle[9]

$$
e_{N}=\max _{0 \leq i \leq N}\left|y_{i}^{N}-y_{2 i}^{2 N}\right|
$$

The maximum absolute error is tabulated in the form of Tables 1-5 for considered examples. The graphs of the solution of the considered examples for different values of delay 
parameter are plotted in Fig. 1-11 to examine the effect of delay on the boundary layer behaviour of the solution.

Example $1\left[5\right.$, p. 275]: $\varepsilon y^{\prime \prime}(\mathrm{x})+\mathrm{y}^{\prime}(\mathrm{x}-\delta)+\mathrm{y}(\mathrm{x})=0$, subject to the interval and boundary conditions $\mathrm{y}(\mathrm{x})=1 ;-\delta \leq \mathrm{x} \leq 0, \mathrm{y}(1)=1$.

Example 2[8, p.700]: $\varepsilon y^{\prime \prime}(\mathrm{x})+0.25 \mathrm{y}^{\prime}(\mathrm{x}-\delta)-\mathrm{y}(\mathrm{x})=0$ subject to the interval and boundary conditions $\mathrm{y}(\mathrm{x})=1 ;-\delta \leq \mathrm{x} \leq 0, \mathrm{y}(1)=-1$.

Example 3[8, p.699]: $\varepsilon y^{\prime \prime}(x)+0.25 y^{\prime}(x-\delta)-y(x)=0$ subject to the interval and boundary conditions $\mathrm{y}(\mathrm{x})=1 ;-\delta \leq \mathrm{x} \leq 0, \mathrm{y}(1)=0$.

Example 4[8, p.707]: $\varepsilon y^{\prime \prime}(\mathrm{x})-\mathrm{y}^{\prime}(\mathrm{x}-\delta)+\mathrm{y}(\mathrm{x})=0$

subject to the interval and boundary conditions $\mathrm{y}(\mathrm{x})=1 ;-\delta \leq \mathrm{x} \leq 0, \mathrm{y}(1)=-1$.

Example 5[4, p.257]: $\varepsilon y^{\prime \prime}(\mathrm{x})-\mathrm{y}^{\prime}(\mathrm{x}-\delta)-\mathrm{y}(\mathrm{x})=0$

subject to the interval and boundary conditions $\mathrm{y}(\mathrm{x})=1 ;-\delta \leq \mathrm{x} \leq 0, \mathrm{y}(1)=-1$.

Table 1. The maximum absolute errors for example 1 with $\delta=0.03$

\begin{tabular}{|c|c|c|c|c|c|}
\hline$\varepsilon$ & $\mathrm{N}=100$ & $\mathrm{~N}=200$ & $\mathrm{~N}=300$ & $\mathrm{~N}=400$ & $\mathrm{~N}=500$ \\
\hline $2^{-1}$ & $8.5448 \mathrm{e}-007$ & $2.1361 \mathrm{e}-007$ & $9.4936 \mathrm{e}-008$ & $5.3409 \mathrm{e}-008$ & $3.4177 \mathrm{e}-008$ \\
\hline $2^{-2}$ & $5.6349 \mathrm{e}-006$ & $1.4090 \mathrm{e}-006$ & $6.2621 \mathrm{e}-007$ & $3.5226 \mathrm{e}-007$ & $2.2544 \mathrm{e}-007$ \\
\hline $2^{-3}$ & $9.4237 \mathrm{e}-006$ & $2.3561 \mathrm{e}-006$ & $1.0472 \mathrm{e}-006$ & $5.8905 \mathrm{e}-007$ & $3.7699 \mathrm{e}-007$ \\
\hline $2^{-4}$ & $5.4815 \mathrm{e}-005$ & $1.3736 \mathrm{e}-005$ & $6.1099 \mathrm{e}-006$ & $3.4362 \mathrm{e}-006$ & $2.1994 \mathrm{e}-006$ \\
\hline $2^{-5}$ & $1.0725 \mathrm{e}-004$ & $2.6826 \mathrm{e}-005$ & $1.1924 \mathrm{e}-005$ & $6.7074 \mathrm{e}-006$ & $4.2928 \mathrm{e}-006$ \\
\hline $2^{-6}$ & 0.0717 & 0.0164 & 0.0072 & 0.0040 & 0.0026 \\
\hline
\end{tabular}

Table 2. The maximum absolute errors for example 2 with $\delta=0.03$

\begin{tabular}{|c|c|c|c|c|c|}
\hline$\varepsilon$ & $\mathrm{N}=100$ & $\mathrm{~N}=200$ & $\mathrm{~N}=300$ & $\mathrm{~N}=400$ & $\mathrm{~N}=500$ \\
\hline $2^{-1}$ & $2.0585 \mathrm{e}-006$ & $5.1461 \mathrm{e}-007$ & $2.2872 \mathrm{e}-007$ & $1.2866 \mathrm{e}-007$ & $8.2338 \mathrm{e}-008$ \\
\hline $2^{-2}$ & $2.0245 \mathrm{e}-006$ & $5.0614 \mathrm{e}-007$ & $2.2496 \mathrm{e}-007$ & $1.2654 \mathrm{e}-007$ & $8.0985 \mathrm{e}-008$ \\
\hline $2^{-3}$ & $2.7779 \mathrm{e}-005$ & $6.9410 \mathrm{e}-006$ & $3.0846 \mathrm{e}-006$ & $1.7351 \mathrm{e}-006$ & $1.1104 \mathrm{e}-006$ \\
\hline $2^{-4}$ & $1.0130 \mathrm{e}-005$ & $2.5332 \mathrm{e}-006$ & $1.1259 \mathrm{e}-006$ & $6.3336 \mathrm{e}-007$ & $4.0510 \mathrm{e}-007$ \\
\hline $2^{-5}$ & $5.5951 \mathrm{e}-004$ & $1.3966 \mathrm{e}-004$ & $6.2050 \mathrm{e}-005$ & $3.4899 \mathrm{e}-005$ & $2.2335 \mathrm{e}-005$ \\
\hline $2^{-6}$ & $1.7087 \mathrm{e}-004$ & $4.2754 \mathrm{e}-005$ & $1.9005 \mathrm{e}-005$ & $1.0691 \mathrm{e}-005$ & $6.8424 \mathrm{e}-006$ \\
\hline $2^{-7}$ & 0.0462 & 0.0116 & 0.0051 & 0.0029 & 0.0019 \\
\hline
\end{tabular}

Table 3. The maximum absolute errors for example 3 with $\delta=0.03$

\begin{tabular}{|c|c|c|c|c|c|}
\hline$\varepsilon$ & $\mathrm{N}=100$ & $\mathrm{~N}=200$ & $\mathrm{~N}=300$ & $\mathrm{~N}=400$ & $\mathrm{~N}=500$ \\
\hline $2^{-1}$ & $1.2359 \mathrm{e}-006$ & $3.0899 \mathrm{e}-007$ & $1.3733 \mathrm{e}-007$ & $7.7249 \mathrm{e}-008$ & $4.9438 \mathrm{e}-008$ \\
\hline $2^{-2}$ & $2.0246 \mathrm{e}-006$ & $5.0615 \mathrm{e}-007$ & $2.2496 \mathrm{e}-007$ & $1.2654 \mathrm{e}-007$ & $8.0986 \mathrm{e}-008$ \\
\hline $2^{-3}$ & $2.9569 \mathrm{e}-006$ & $7.3925 \mathrm{e}-007$ & $3.2856 \mathrm{e}-007$ & $1.8482 \mathrm{e}-007$ & $1.1828 \mathrm{e}-007$ \\
\hline $2^{-4}$ & $1.0130 \mathrm{e}-005$ & $2.5332 \mathrm{e}-006$ & $1.1259 \mathrm{e}-006$ & $6.3334 \mathrm{e}-007$ & $4.0534 \mathrm{e}-007$ \\
\hline $2^{-5}$ & $4.2189 \mathrm{e}-005$ & $1.0552 \mathrm{e}-005$ & $4.6902 \mathrm{e}-006$ & $2.6383 \mathrm{e}-006$ & $1.6885 \mathrm{e}-006$ \\
\hline $2^{-6}$ & $1.7087 \mathrm{e}-004$ & $4.2754 \mathrm{e}-005$ & $1.9005 \mathrm{e}-005$ & $1.0691 \mathrm{e}-005$ & $6.8424 \mathrm{e}-006$ \\
\hline $2^{-7}$ & $6.8796 \mathrm{e}-004$ & $1.7229 \mathrm{e}-004$ & $7.6599 \mathrm{e}-005$ & $4.3092 \mathrm{e}-005$ & $2.7580 \mathrm{e}-005$ \\
\hline
\end{tabular}

Table 4. The maximum absolute errors for example 4 with $\delta=0.03$

\begin{tabular}{|c|c|c|c|c|c|}
\hline$\varepsilon$ & $\mathrm{N}=100$ & $\mathrm{~N}=200$ & $\mathrm{~N}=300$ & $\mathrm{~N}=400$ & $\mathrm{~N}=500$ \\
\hline $2^{-1}$ & $1.1690 \mathrm{e}-006$ & $2.9231 \mathrm{e}-007$ & $1.2992 \mathrm{e}-007$ & $7.3081 \mathrm{e}-008$ & $4.6775 \mathrm{e}-008$ \\
\hline $2^{-2}$ & $2.5483 \mathrm{e}-006$ & $6.3707 \mathrm{e}-007$ & $2.8314 \mathrm{e}-007$ & $1.5927 \mathrm{e}-007$ & $1.0192 \mathrm{e}-007$ \\
\hline $2^{-3}$ & $1.0292 \mathrm{e}-005$ & $2.5732 \mathrm{e}-006$ & $1.1437 \mathrm{e}-006$ & $6.4333 \mathrm{e}-007$ & $4.1173 \mathrm{e}-007$ \\
\hline $2^{-4}$ & $5.2798 \mathrm{e}-005$ & $1.3210 \mathrm{e}-005$ & $5.8719 \mathrm{e}-006$ & $3.3031 \mathrm{e}-006$ & $2.1140 \mathrm{e}-006$ \\
\hline $2^{-5}$ & $1.8566 \mathrm{e}-004$ & $4.6473 \mathrm{e}-005$ & $2.0659 \mathrm{e}-005$ & $1.1621 \mathrm{e}-005$ & $7.4380 \mathrm{e}-006$ \\
\hline $2^{-6}$ & $5.3884 \mathrm{e}-004$ & $1.3484 \mathrm{e}-004$ & $5.9943 \mathrm{e}-005$ & $3.3721 \mathrm{e}-005$ & $2.1582 \mathrm{e}-005$ \\
\hline $2^{-7}$ & 0.0014 & $3.4603 \mathrm{e}-004$ & $1.5389 \mathrm{e}-004$ & $8.6588 \mathrm{e}-005$ & $5.5422 \mathrm{e}-005$ \\
\hline
\end{tabular}

Table 5. The maximum absolute errors for example 5 with $\delta=0.03$

\begin{tabular}{|c|c|c|c|c|c|}
\hline$\varepsilon$ & $\mathrm{N}=100$ & $\mathrm{~N}=200$ & $\mathrm{~N}=300$ & $\mathrm{~N}=400$ & $\mathrm{~N}=500$ \\
\hline $2^{-1}$ & $1.5692 \mathrm{e}-006$ & $3.9229 \mathrm{e}-007$ & $1.7435 \mathrm{e}-007$ & $9.8067 \mathrm{e}-008$ & $6.2765 \mathrm{e}-008$ \\
\hline $2^{-2}$ & $3.3241 \mathrm{e}-006$ & $8.3084 \mathrm{e}-007$ & $3.6925 \mathrm{e}-007$ & $2.0770 \mathrm{e}-007$ & $1.3292 \mathrm{e}-007$ \\
\hline $2^{-3}$ & $9.7800 \mathrm{e}-006$ & $2.4452 \mathrm{e}-006$ & $1.0867 \mathrm{e}-006$ & $6.1130 \mathrm{e}-007$ & $3.9123 \mathrm{e}-007$ \\
\hline $2^{-4}$ & $3.3415 \mathrm{e}-005$ & $8.3551 \mathrm{e}-006$ & $3.7135 \mathrm{e}-006$ & $2.0889 \mathrm{e}-006$ & $1.3369 \mathrm{e}-006$ \\
\hline $2^{-5}$ & $1.0770 \mathrm{e}-004$ & $2.6936 \mathrm{e}-005$ & $1.1973 \mathrm{e}-005$ & $6.7348 \mathrm{e}-006$ & $4.3103 \mathrm{e}-006$ \\
\hline $2^{-6}$ & $3.2451 \mathrm{e}-004$ & $8.1217 \mathrm{e}-005$ & $3.6104 \mathrm{e}-005$ & $2.0310 \mathrm{e}-005$ & $1.2999 \mathrm{e}-005$ \\
\hline $2^{-7}$ & $9.1833 \mathrm{e}-004$ & $2.3014 \mathrm{e}-004$ & $1.0233 \mathrm{e}-004$ & $5.7570 \mathrm{e}-005$ & $3.6848 \mathrm{e}-005$ \\
\hline
\end{tabular}




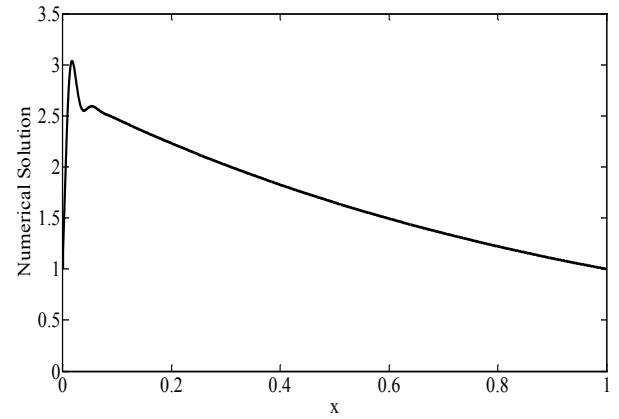

Figure 1. The numerical solution of example 1 with $\varepsilon=0.01$ and $\delta=0.007$.

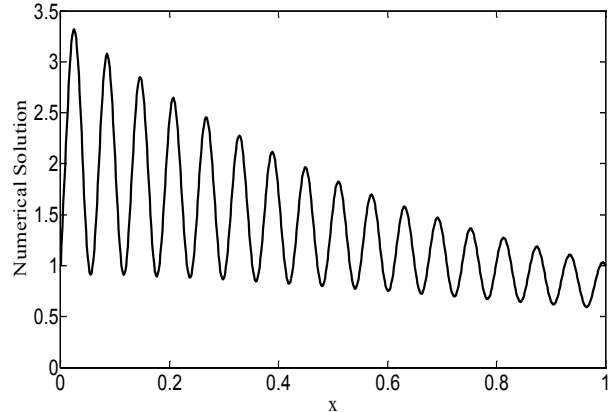

Figure 2. The numerical solution of example 1 with $\varepsilon=0.01$ and $\delta=0.015$.

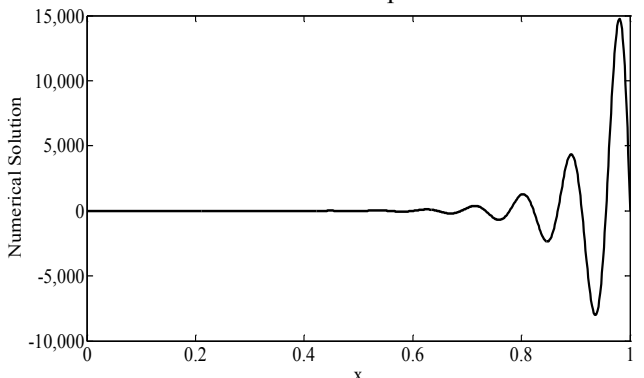

Figure 3. The numerical solution of example 1 with $\varepsilon=0.01$ and $\delta=0.025$.

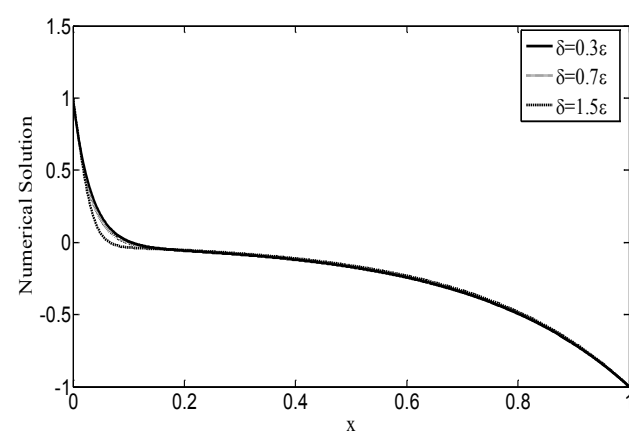

Figure 4. The numerical solution of example 2 with $\varepsilon=0.01$ for different values of $\delta$.

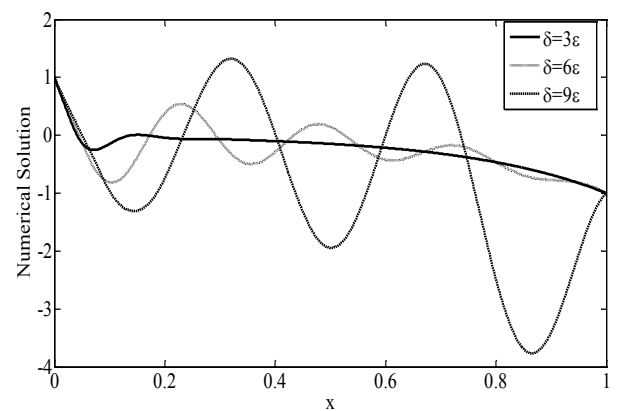

Figure 5. The numerical solution of example 2 with $\varepsilon=0.01$ for different values of $\delta$.

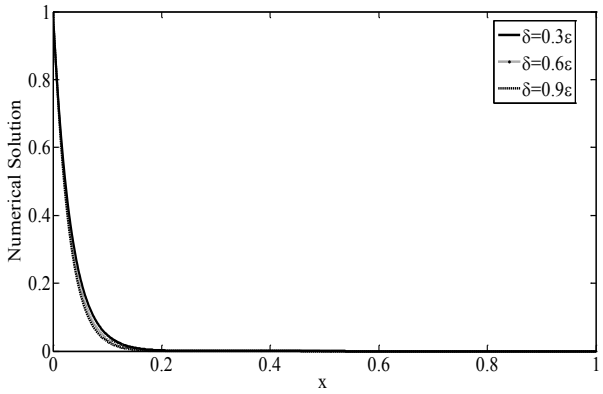

Figure 6. The numerical solution of example 3 with $\varepsilon=0.01$ for different values of $\delta$.

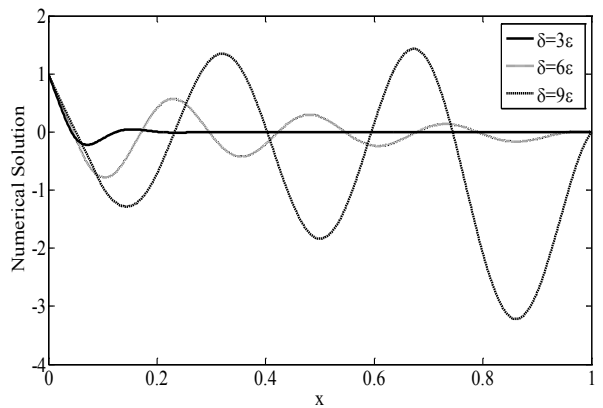

Figure 7. The numerical solution of example 3 with $\varepsilon=0.01$ for different values of $\delta$.

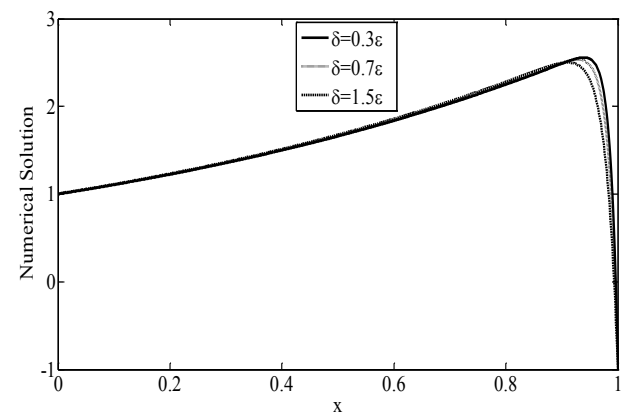

Figure 8. The numerical solution of example 4 with $\varepsilon=0.01$ for different values of $\delta$.

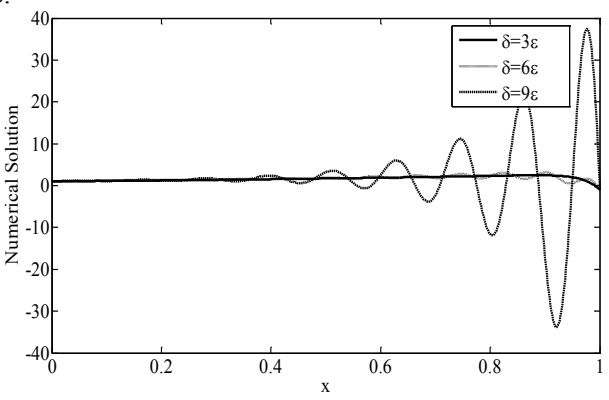

Figure 9. The numerical solution of example 4 with $\varepsilon=0.01$ for different values of $\delta$.

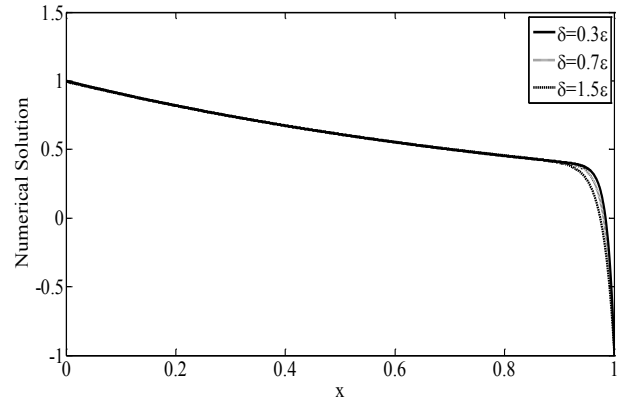

Figure 10. The numerical solution of example 5 with $\varepsilon=0.01$ for different values of $\delta$. 


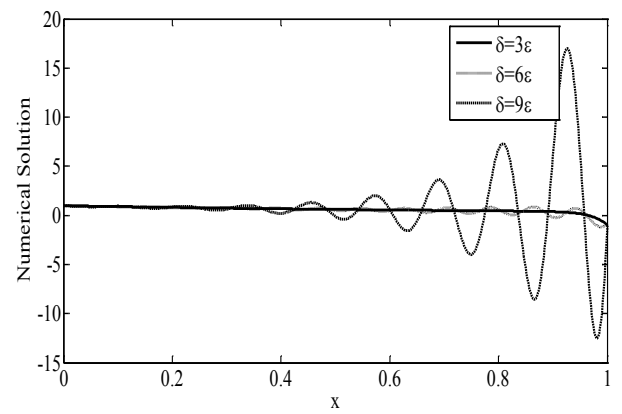

Figure 11. The numerical solution of example 5 with $\varepsilon=0.01$ for different values of $\delta$.

\section{Conclusions}

A fourth order finite difference method is developed for solving singularly perturbed differential difference equations. The proposed finite difference method works nicely when the delay parameter is smaller or bigger to perturbation parameter. The truncation error of the finite difference scheme is calculated. It is observed that when the delay parameter is smaller than the perturbation parameter, the layer behaviour is maintained. As the delay increases, thickness of the layer decreases in the case when the solution exhibits layer behaviour on the left side while in the case of the right side boundary layer, it increases. And for the delay parameter greater than the perturbation parameter, it is observed that the layer behaviour of the solution is no longer maintained and the solution exhibits oscillatory behaviour. Also when the delay further increases the oscillations previously confined to the layer region are extended throughout the interval $[0,1]$. From the results, it can be observed that as the grid size $\mathrm{h}$ decreases, the maximum absolute errors decrease, which shows the convergence to the computed solution. On the basis of truncation error, as well as the results of number of computational examples, it is concluded that the present method offers significant advantage for the linear singularly perturbed differential difference equations.

\section{ACKNOWLEDGEMENTS}

The authors wish to thank the Department of Science \& Technology, Government of India, for their financial support under the project No. SR/S4/MS: 598/09.

\section{REFERENCES}

[1] Y. Kuang, Delay Differential equations with applications in population dynamics, Academic Press, 1993

[2] L.E. El'sgol'ts, Qualitative Methods in Mathematical Analyses, Translations of Mathematical Monographs 12, American mathematical society, Providence, RI, 1964
[3] R.B. Stein, A theoretical analysis of neuronal variability, Biophysical Journal 5 (1965) 173-194

[4] C.G. Lange, R.M. Miura, Singular perturbation analysis of boundary-value problems for differential difference equations. V. Small shifts with layer behavior, SIAM Journal on Applied Mathematics 54 (1994) 249-272

[5] C.G. Lange, R.M. Miura, Singular perturbation analysis of boundary-value problems for differential difference equations. VI. Small shifts with rapid oscillations, SIAM Journal on Applied Mathematics 54 (1994) 273-283

[6] Mohan K. Kadalbajoo, Kapil K. Sharma, Numerical analysis of singularly perturbed delay differential equations with layer behavior, Applied Mathematics and Computation 157 (2004) 11-28

[7] Mohan K. Kadalbajoo, Kapil K. Sharma, Parameter-Uniform fitted mesh method for singularly perturbed delay differential equations with layer behavior, Electronic Transactions on Numerical Analysis, Vol. 23(2006) 180-201

[8] Mohan K. Kadalbajoo, Kapil K. Sharma, A numerical method based on finite difference for boundary value problems for singularly perturbed delay differential equations, Applied Mathematics and Computation 197 (2008) 692-707

[9] E.P. Doolan, J.J.H. Miller, W.H.A. Schilders, Uniform Numerical methods for problems with initial and boundary layers, Boole Press, Dublin, 1980

[10] E.A. Boqucz, J.D.A. Walker, Fourth order finite difference methods for two-point boundary value problems, I.M.A. Journal of Numer. Anal., 4 (1984) 69-82

[11] Mohan K. Kadalbajoo, Devendra Kumar, A computational method for singularly perturbed nonlinear differential-difference equations with small shift, Applied Mathematical Modelling Vol. 34 (2010) 2584-2596

[12] Gabil M. Amiraliyev, Erkan Cimen, Numerical method for a singularly perturbed convection-diffusion problem with delay, Applied Mathematics and Computation Vol. 216 (2010) 2351-2359

[13] Mohan.K. Kadalbajoo, Devendra Kumar, Fitted mesh B-spline collocation method for singularly perturbed differential-difference equations with small delay, Applied Mathematics and Computation Vol. 204 (2008) 90-98

[14] Mohan K. Kadalbajoo, V.P. Ramesh, Hybrid method for numerical solution of singularly perturbed delay differential equations, Applied Mathematics and Computation Vol. 187 (2007) 797-814

[15] Jugal Mohapatra, Srinivasan Natesan, Uniformly convergent numerical method for singularly perturbed differential-difference equation using grid equidistribution, International Journal for Numerical Methods in Biomedical Engineering, Vol. 27, Issue 9, (2011) 1427-1445

[16] Fox L, The numerical solution of two-point boundary value problems in ordinary differential equations, Oxford university press, London, 1957 